\title{
EO System Concepts in the Littoral
}

\author{
Piet B.W. Schwering, Sebastiaan P. van den Broek, Miranda van Iersel
}

TNO Defence, Security and Safety, P.O. Box 96864, NL-2509 JG The Hague, Netherlands, piet.schwering@tno.nl

\begin{abstract}
In recent years, operations executed by naval forces have taken place at many different locations. At present, operations against international terrorism and asymmetric warfare in coastal environments are of major concern. In these scenarios, the threat caused by pirates on-board of small surface targets, such as jetskis and fast inshore attack crafts, is increasing. In the littoral environment, the understanding of its complexity and the efficient use of the limited reaction time, are essential for successful operations. Present-day electro-optical sensor suites, also incorporating Infrared Search and Track systems, can be used for varying tasks as detection, classification and identification. By means of passive electrooptical systems, infrared and visible light sensors, improved situational awareness can be achieved. For long range capability, elevated sensor masts and flying platforms are ideally suited for the surveillance task and improve situational awareness. A primary issue is how to incorporate new electro-optical technology and signal processing into the new sensor concepts, to improve system performance. It is essential to derive accurate information from the high spatialresolution imagery created by the EO sensors. As electro-optical sensors do not have all-weather capability, the performance degradation in adverse scenarios must be understood, in order to support the operational use of adaptive sensor management techniques. In this paper we discuss the approach taken at TNO in the design and assessment of system concepts for future IRST development. An overview of our maritime programme in future IRST and EO system concepts including signal processing is presented.
\end{abstract}

Keywords: Electro-optical systems, system concepts, image enhancement, detection, classification.

\section{INTRODUCTION}

Present-day naval operations take place in coastal environments as well as narrow straits all over the world. Coastal environments around the world are exhibiting a number of threats to naval forces. In particular, a large number of asymmetric threats can be present in environments with cluttered backgrounds as well as rapidly varying atmospheric conditions. In these conditions the threat contrast may be low and varying, and the amount of background clutter can be severe. In coastal environments electro-optical means of detection and classification should be optimized in order to have more time to act against threats. In particular the assessment of classification means is an important issue.

Electro-optical sensor suites can be used for diverse tasks as detection, classification and identification. By means of passive electro-optical systems, such as infrared and visible light sensors, improved situational awareness can be achieved. For long range capability elevated sensor masts and flying platforms are ideally suited for the surveillance task and improve the operational picture. A primary issue is how to incorporate new electro-optical technology and signal processing into the new system concepts. Of great importance is the derivation of information from the high spatial-resolution imagery created by the sensors. As electro-optical sensors do not have all-weather capability, the performance degradation in adverse scenarios should be understood, to support the operational use of adaptive sensor management techniques.

At present TNO is investigating Electro-Optical sensor systems to extend the scientific and technological knowledge and models, in the field of the next generation staring electro-optical sensor systems:

- to support situational awareness and operational picture

Infrared Technology and Applications XXXIII, edited by Bjørn F. Andresen, Gabor F. Fulop, Paul R. Norton, Proc. of SPIE Vol. 6542, 654230, (2007) · 0277-786X/07/\$18 · doi: 10.1117/12.719217 
- for detection and classification

- to support development and procurement of sensors

This knowledge is already being applied during the execution of operational tasks, in particular in littoral environments.

Apart from the naval scenario, algorithms have been developed for detection of threats in land scenarios. For detection the algorithms make use of different kinds of target features, such as hot spots, motion, and structure. Algorithms for adaptive sensor management are being tested making use of live recordings from naval operations. Incorporation into new types of EO system concepts, based on infrared and visible light systems as well as integrated systems, is foreseen.

In Figure 1 we present a few typical examples of present-day threats: small surface vessels and jetskis. Note the high intensity levels of the coastal background, as well as the higher intensities of the object's wake at sea. The first one may hinder detection, and the second one can also support the detection process.
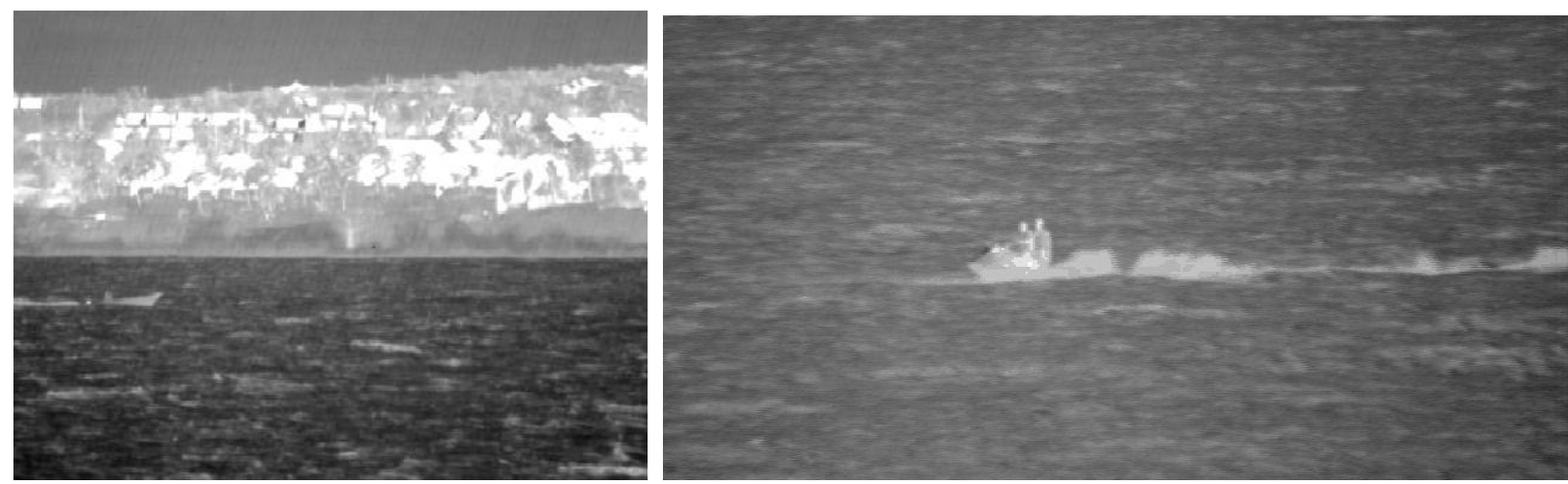

Figure 1: Examples of infrared images of potential coastal threats, a small vessel and jetski in a warm coastal environment.

In this paper we present our research programme at TNO to investigate methods for application in future EO sensor systems for detection and classification of small objects at sea. We will discuss system concepts, the suite of algorithms required, the system block diagram, as well as some first examples of image enhancement in the maritime environment in particular to counter turbulence. We also discuss the topics on classification, adaptive sensor management, and applications of contrast enhancement. We finish with the conclusions.

\section{PROGRAMME OVERVIEW}

Of primary importance is the real-time assessment of threats. Therefore autonomous techniques for detection, classification and identification of threats, remains a major task of study. Especially the amount of clutter and changing operational conditions have an impact on the threats' contrast and complicate the distinction between neutral and hostile units. The detection techniques should be robust and allow for only few false alarms. Furthermore, the assessment of the system performance gain, due to the application of new electro-optical techniques, should be accurately quantified. The emphasis is on improved classification methods. Our plan is to demonstrate these techniques in a relevant environment at a Technology Readiness Level up to 5, to fully support future industrial applications making use of these techniques.

The present research topics of the programme are focused on:

- improving performance in detection, classification and false alarm reduction in the use of staring EO-systems and its image processing and detection technology

- system concept analysis for application in elevated platforms for short range platform protection in littoral, land and airborne scenarios

- improving performance in situational awareness, operational picture, image processing and adaptive sensor management, and integration in the combat management system 
- analysis and road mapping of the operational use of new electro-optical system technologies, such as developments in the fields of optics and detectors

- application of image processing techniques in staring sensors, in particular for image stitching, resolution and noise improvement, in moving platforms

- autonomous techniques for detection, classification, tracking of small surface targets

- (semi-)autonomous classification techniques, including clustering, segmentation, feature extraction and pattern recognition

- application of the Triangle Orientation Discrimination method for assessing the performance gain of image improvement methods and autonomous systems

\section{SYSTEM CONCEPTS}

The EO-system should be designed with the objective that it should be able to detect and classify small surface targets in maritime environments. The main operational task is situational awareness. The most important technical parameters in this programme are detection and classification processing.

This new type of Infrared Search and Track (IRST) system, combining search with classification, must therefore have a large field of regard (FOR), high resolution (or instantaneous field of view IFOV) and high sensitivity (or low noise equivalent temperature difference NETD). The system figure of merit is then a combination of these three parameters. In general the requirements will be for IRST, IR cameras and visible light camera systems. In this sense it can be considered as a Multi-Function IRST. The system concept consists of two main parts: The sensor head and the data processing.

\subsection{Sensor requirements}

The basis system tasks are detection and classification. Detection must be performed over the entire FOR. The detection task is normally accompanied by a tracking stage, in which the classification process can be supportive. This means that tracking and classification must be accomplished anywhere in the FOR, but only with locally defined high resolution imagery. Hence only at the area of interest high resolution is required. Although sensitivity is mainly a requirement for the detection stage it is also important for classification.

For different application areas different system approaches can be taken. The present study is aiming for ship-based platforms. We assume EO sensors in a mast at approximately $20 \mathrm{~m}$ height. Application areas for sensors in land based masts or lifted by air platforms are studied in terms of spin-off of these concepts. Operations during day and night must be supported. For the scenario application, the aspects that concern us the most at first are the threat and target optical characteristics and the ways of modelling these in system evaluation. In particular detailed knowledge of the targetbackground interaction can be of great importance for accurate performance predictions.

Our objective for detection and classification of small surface targets is for mid-range $(5-10 \mathrm{~km})$, while allowing for close-range $(0-5 \mathrm{~km})$ operation. It is assumed that for applications such as missile defence, also the horizon search will be sufficient. Investigation of top attacks is not considered here. Therefore the FOR must be from a few degrees above the horizon to several degrees below the horizon. It is envisaged that day-and-night capability is required, allowing for countering the negative effects of degradation by adverse weather conditions.

The basic required parameters at system level are defined to be:

- FOR (field of regard) and FOV (field of view),

- IFOV (instantaneous field of view),

- NETD (noise equivalent temperature difference) and NEI (noise equivalent irradiance),

- FAR (false alarm and false classification rate).

The combined sensitivity and resolution requirements are given in the MRTD/MRC requirements.

\subsection{Multi-Function IRST}

For the typical small surface target a temperature contrast of $2 \mathrm{~K}$ is assumed at present with a dimension of $6 \times 2 \times 0.5$ $\mathrm{m}(l \times w \times h)$. Fluctuation of target contrast, as described in Schwering $(2006)^{1}$, can be dominating the detection of the 
small vessel as wake and other surface disturbance may be over $100 \%$ of the signal in strength and in size. For threat analysis the physical parameters that determine the optical characteristics will need to be defined in detail, thus describing the hard body, in dimensions, shape, hot spots and intensity contrast, as well as the soft body due to surface displacements caused by wakes etc. Hence system requirements are still under development.

Within previous projects a number of system concepts have already been defined. The basic concepts of infrared search and track are:

- Scanning system concepts, consisting of a single scanning system,

- Staring system concepts, consisting of (a set of) staring cameras,

- Hybrid system concepts, consisting of a combination of staring and scanning sensors (step-stare).

Some analysis in these concepts was done in the previous projects EUCLID RTP8.2 $2^{2}$ and SURFER ${ }^{3}$. We have now looked into ways of applying these technologies for detection and classification of small surface targets.

New system concepts under development at industry were presented at the conference on Passive Sensing and Targeting $2006^{4}$. A further topic of interest is the use of separate Track and Recognise Heads (TRH). These can consist of multiple band cameras, high spatial and temporal resolution cameras, lasers, hyperspectral sensors, etc. that provide additional information to classify the target as threat. Mirador (Thales) can be considered as a typical example of a TRH. In the course of the programme, lessons learned from electro-optical sensors have been obtained from discussions and interactions with RNLN Mirador operators.

Basic design inputs are related to the interaction with the platform, these parameters are determined by:

- Position and height on the platform,

- Stabilisation of the sensor,

- System cost.

The application of system concepts can be different. The location can be on the main ship, on specific small sensor ships or floating, airborne, and for instance also distributed on different platforms. Additionally, hand-held electrooptical systems are of interest for new developments in littoral operations. At present, in our studies combinations of uncooled infrared, cooled infrared and visible light cameras is under investigation.

The future IRST system must be able to detect and classify targets. This task also means sufficient clutter rejection. The system will consist of two main components

- Sensor head,

- Processing unit.

The sensor head consists of the following major components: optics, detector, cooling and stabilisation.

The processing unit consists of both hardware and software. The main areas are the front-end electronics, image enhancement, detection process, classification and sensor management processing. In this process the tracking stage can be incorporated in the detection or classification stage.

\subsection{System trade-offs}

There are many choices for upgrading the systems. Therefore trade off must be made to derive to an optimum choice for the required system. The main system trade-offs are the following.

- Staring vs. scanning systems

- Field of view vs. spatial resolution

- Frame rate vs. integration time

- Cooled vs. uncooled sensors

- Signal, image and data processing vs. sensor head

- Amount of automatic classification processing vs. human-in-the-loop

Finally, selected system trade-offs need to be compensated by additional signal processing. Additionally other new technologies may be applicable in extra system trade-offs. New technologies, such as multicamera arrays, turbulence correction methods and wavefront coding, are being investigated. The main constraint in this trade-off process will be the performance to cost ratio in the operational scenarios. 


\section{DATA PROCESSING ALGORITHMS}

Of major interest in development of automatic detection and classification systems is the signal processing. The signal processing determines the quality of the imagery, the detection and clutter rejection capability, as well the classification probabilities. In Figure 2 we present a preliminary system block diagram of the system processing concept of our system. The model overview of the figure shows system areas of image enhancement pre-processing, detection, classification and sensor management. For reasons of clarity only a single (search) camera is specified in this figure. In reality additional other wave band cameras (e.g. TRH) will be used to support the classification process.

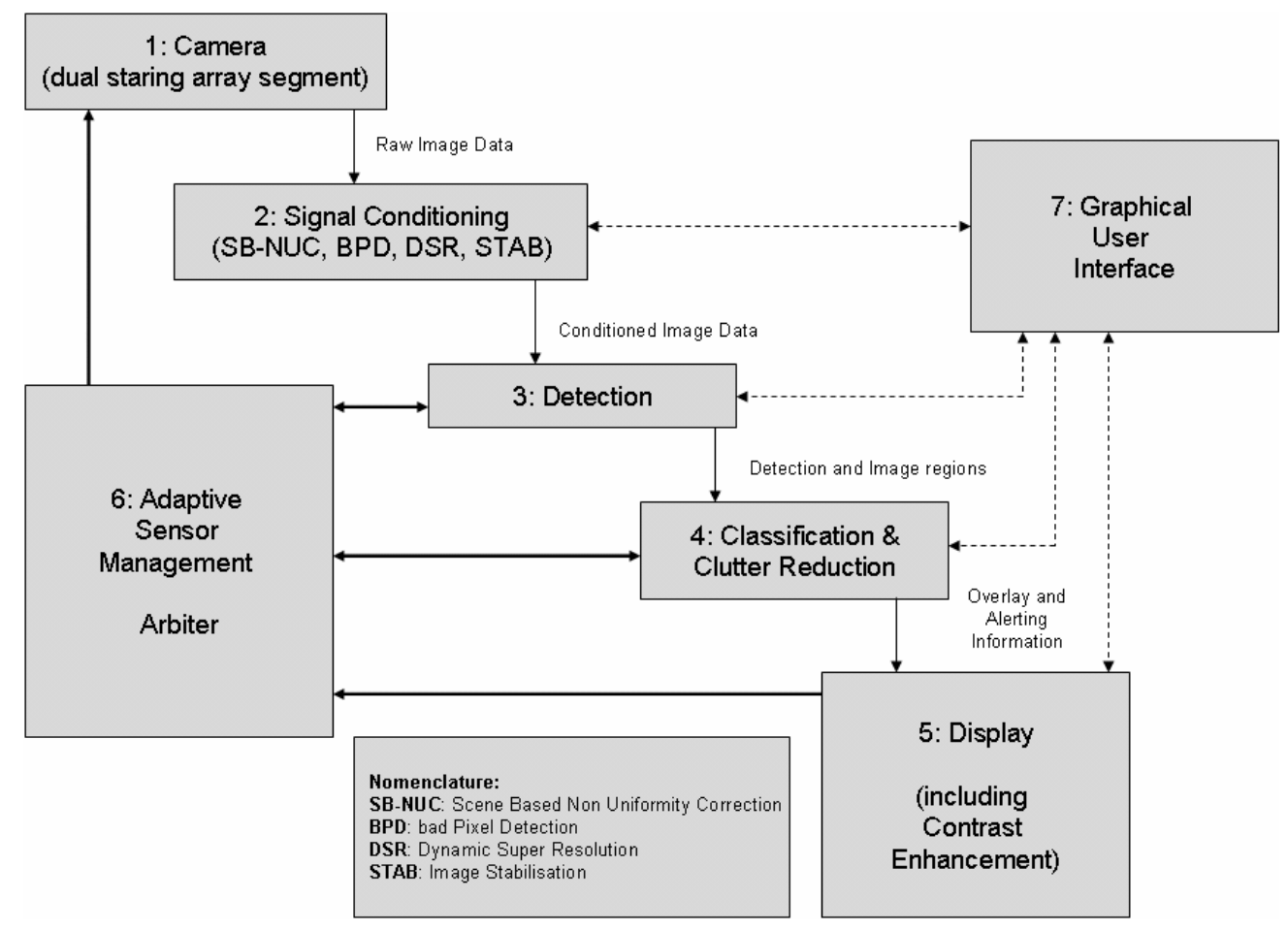

Figure 2: Block diagram of proposed integration of the algorithms, based on a search channel.

The goal of the programme is to use and adapt existing software for image enhancement, detection, classification and sensor management and to make a robust real-time maritime demonstrator, which supplies enhanced imagery for the operator and automatically detect small surface targets, such as jetskis and fast inshore attack crafts.

\subsection{Signal conditioning}

An assessment has been made how SURFER detection algorithms ${ }^{3}$ and the TNO camera signal conditioning algorithms $\mathrm{s}^{5}$ can efficiently co-operate in an integrated way. Camera signal conditioning algorithms cover image enhancement and SURFER algorithms are developed for detection and object characterisation. The processes classification, clutter reduction and adaptive sensor management, that automatically select the most interesting image, are under development. In Figure 2 the pipe line of the SURFER (block 3) and signal conditioning processing (block 2) entities are shown. Optionally, when the adaptive sensor management (block 6) is activated, automatic invocation of contrast enhancement or the adaptation of thresholds can become possible. At present the interfacing between the different modules will be harmonised and the communication will be determined. It may be necessary to perform processing of the combined solution (signal conditioning and SURFER detection and object characterisation) on more than one platform. Therefore, internet protocol communication between some of the blocks will be considered.

For improved classification over long ranges turbulence correction is essential. The effects of turbulence in the atmosphere can become problematic when one is interested in obtaining a highly detailed image. When safeguarding harbours such a detailed image is required to be able to tell if there are any threats in the vicinity. The turbulence in the 
atmosphere can distort the images, making it hard to see details. There are several possibilities to correct for the effects of turbulence in images. We have looked at the possibility of image restoration after the images are recorded. Especially after image stabilisation turbulence is dominating the image sequence and limits classification.

We investigated how the standard image enhancement suite of $\mathrm{TNO}^{5}$ can be extended with turbulence compensation. This image enhancement suite contains dynamic super-resolution (DSR), local adaptive contrast enhancement (LACE), image stabilisation and moving object detection. In Figure 3 an input image from the image sequence is shown together with an output image obtained after applying turbulence compensation. The amount of detail visible in the images has grown and the scintillations have disappeared. Note that the effect of turbulence is clearer in a movie than in a single image. Moving objects like birds are detected and preserved in the output images. For slow moving objects the smearing effect due to temporal integration is negligible and these objects are well visible in the output images.

Using turbulence compensation in combination with the TNO image enhancement suite on images with turbulence gives stable unblurred and detailed images in which small and moving objects are preserved. Our technique for turbulence correction, as exemplified in Figure 3 below, improves the situational awareness, by using stabilisation and creating a sharper image of the ship at $12.5 \mathrm{~km}$ distance.
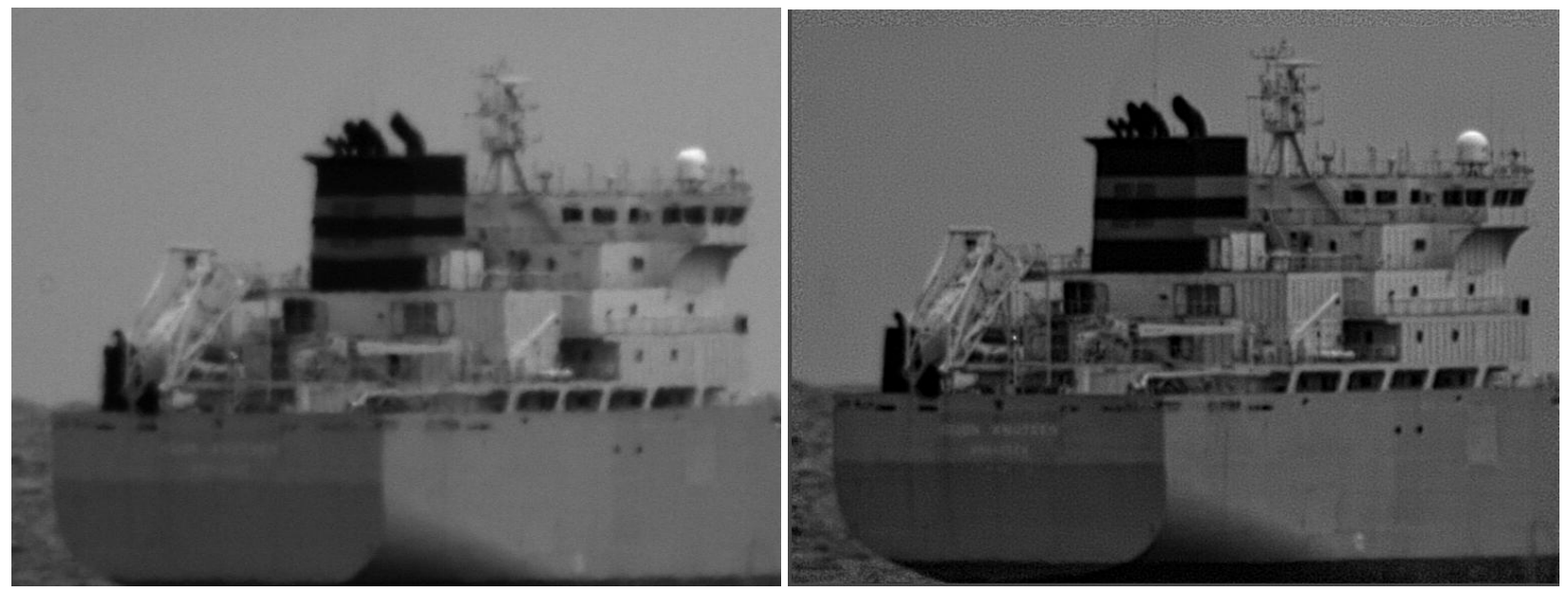

Figure 3: $\quad$ Originally recorded visible light image (left) and image processed with turbulence corrector (right). The ship's range is $12.5 \mathrm{~km}$. Images were recorded with the TNO SPITS ${ }^{6}$ system.

\subsection{Detection}

The detection process will be investigated in the near future. The present ideas are that there will be two parallel detectors, one detector for the hot spots and one for moving targets (Schutte et al. ${ }^{5}$ ). It is foreseen to use the existing TNO tracker ${ }^{7}$ (MT3 or M6T) to do temporal correlation and confirmations of potential detections. A description of the SURFER hot spot detection algorithm is presented in van den Broek et al. ${ }^{3}$. An important stage in the detection preprocessing is the background removal, especially in close-by situations with large amounts of sky reflections and other variations (such as white caps) in the background levels.

\subsection{Classification and Clutter rejection}

The design of the classification process, as it will be implemented in combination with other processing in the demonstrator, should describe several tasks. Examples are clutter reduction where simple features are used to classify between clutter and non-clutter detections, resulting in lower false alarm rates for comparable detection probability. Tracking may further reduce false alarms. Two approaches are foreseen in the programme at present:

- Information enhancement by using simple features (such as size) and specifying extra information to an operator, possibly including general classes (large/medium/small, or containership, fishing vessel, ...)

- Aided target recognition: After a selection based on earlier steps, more complicated features (such as possibly profiles, input from an operator) are used to specify a few possible types of ships, with reliability. 
The inputs for classification are detections with information from which features can be derived. Clutter reduction can be considered as a form of classification, which is used early in the process to limit the processing load for further, more complicated methods. Tracking adds the possibility to look at changes of features in time, or to determine features more accurately. It also provides information such as velocity. Classification may be done in steps, for example repeating several feature extraction and classification steps. In this way more complicated steps, need not be applied to all detected objects. The output of the process will often be object information or class information being presented to an operator. A literature study (and overview of results of previous projects) is done at TNO Human Factors for this project. It will be used to guide the further design and implementation of the classification process. It provides a description of existing systems, and results from research on the use of aided target recognition.

Features to be used may be obvious, such as size related features. Others were derived in other projects (such as features based on intensity variation in SURFER ${ }^{3}$ ), or are common choices in pattern recognition (such as profiles, or moments). A start was made with examining different features, using a limited set of available images. Figure 4 shows some examples of images with segmentation masks that were used to derive some basic features. More complicated features, such as moments are also being looked at.

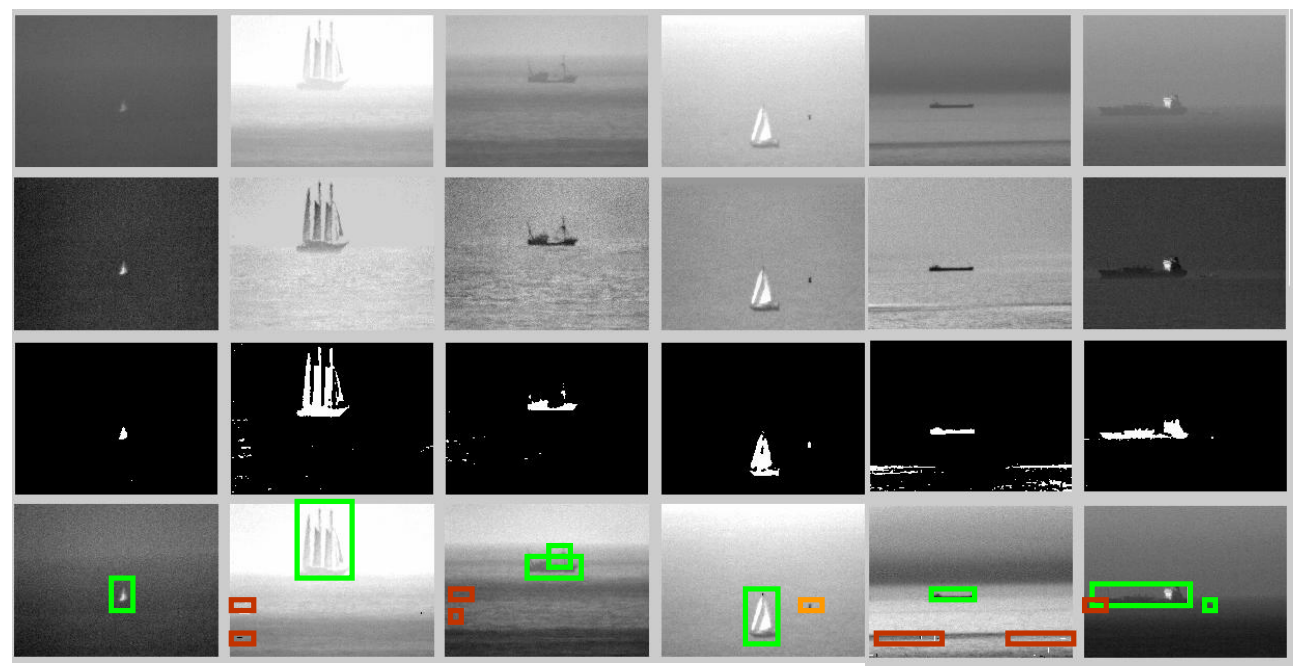

Figure 4: $\quad$ Examples of segmentation of objects and false alarms due to clutter, as basis for feature extraction. From top to bottom, the original (visible light) image, background corrected image (SURFER algorithm), object masks, and tracking results. Images were recorded with the TNO SPITS ${ }^{6}$ system.

Figure 5 shows an example of features related to ships and clutter. These features (computed from contrast variations on and next to the object), were derived in the project SURFER and show clearly that clutter reduction is possible by posing a limit on such features.

For classification, a database is needed containing information relating features to specific classes. For clutter reduction this is simple, but for using more classes, defining the database is much more complex. Not only does it require much data to fill the database, but separating classes may get difficult for other reasons, as shown in Figure 6. The left figure shows 3-sigma covariance level for two features of three different objects (three shapes), in a range of 1 to 8 kilometres, and aspect angles from 0 to 90 degrees. The classes clearly overlap. The right figure shows only values for aspect angles 75-90 degrees and for ranges 1-4 and 5-8 km separately. The distribution is now more compact. This shows that classification between these objects is greatly helped if an estimation of aspect angle (and distance) is available (for example from a radar track), so comparing features can be done for this subset of classes. This will however pose some questions about availability of data to build a database that will be further examined. 

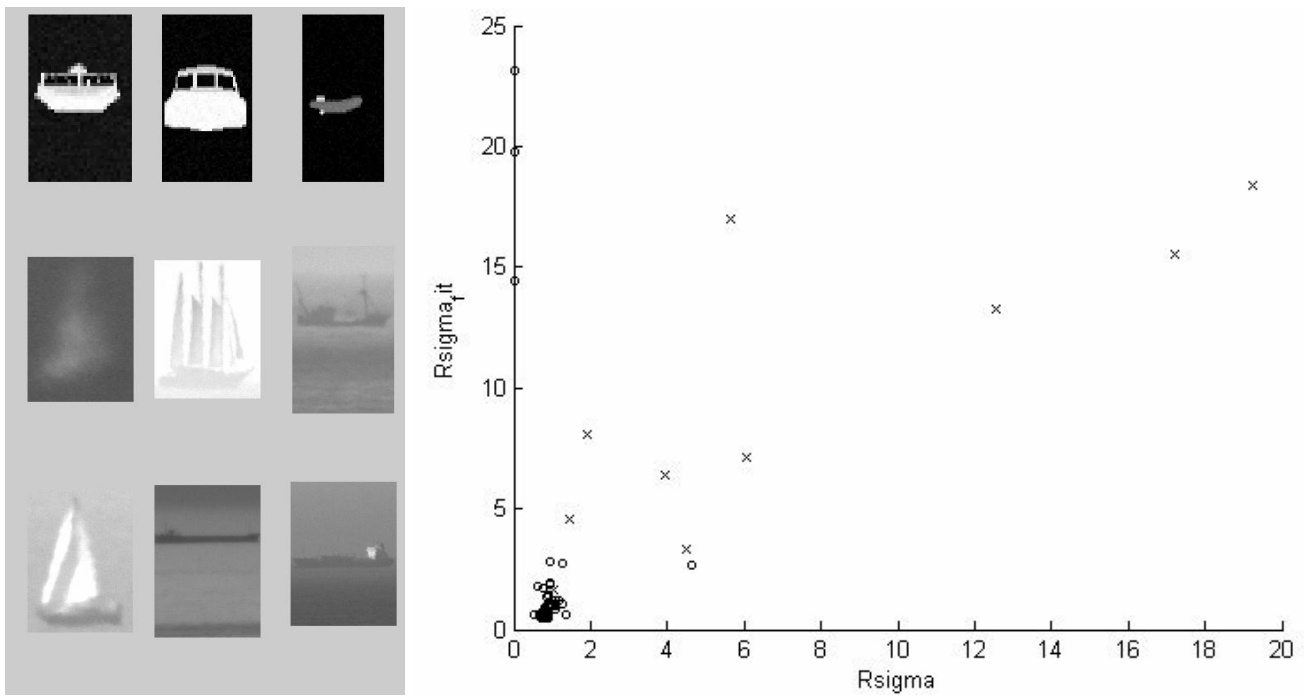

Figure 5: $\quad$ Example of features usable for clutter reduction. The crosses are alarms related to the 9 objects presented at the left, circles are alarms due to clutter. The three top images are simulated.

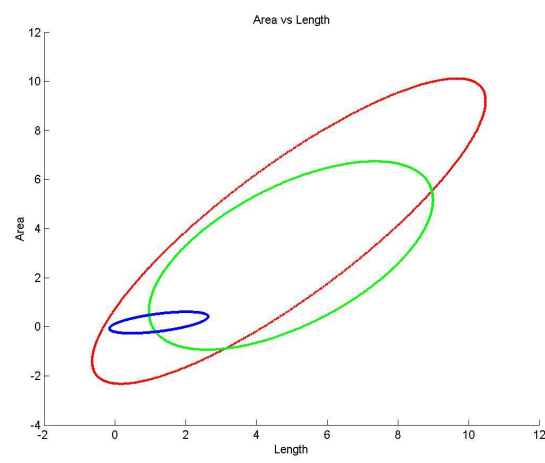

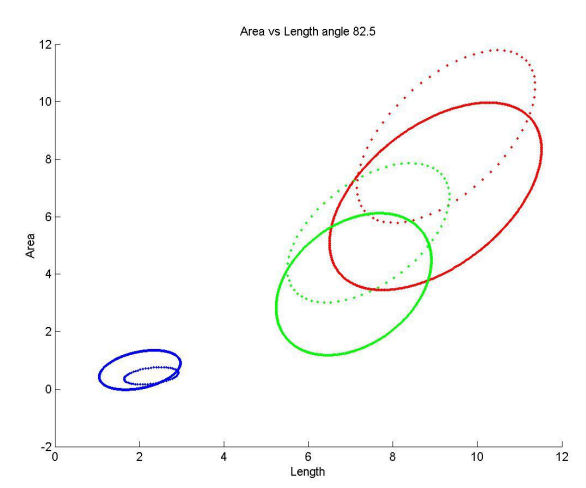

b)

Figure 6: $\quad$ Distribution of two features (detection width and area) for three simulated objects (see top of Figure 5). a) all ranges and aspect angles b) limited range (1-4 km solid, 5-8 km dotted) and aspect angles (75-90 degrees). The 3-sigma covariance of the features is presented.

\subsection{Contrast enhancement}

During several experiments in a naval environment we have tested the Local Adaptive Contrast Enhancement (LACE) module on-board naval ships. These experiments took place in a harbour environment and several passages though narrow straits. Some results are presented below. In the Figures 7-9 the left images are the original image from the sensor. After processing with the LACE module the right image shows the effect of increased contrast on these images. Examples are from the MWIR infrared, LLL-TV as well as visible light imagery. The results show that clearly situational awareness is improved in these conditions. This is visible in the imagery of the figures by the presence of more details in the background. Structure information is better visible in the processed imagery, hence improving operator aided classification and classification ranges. 


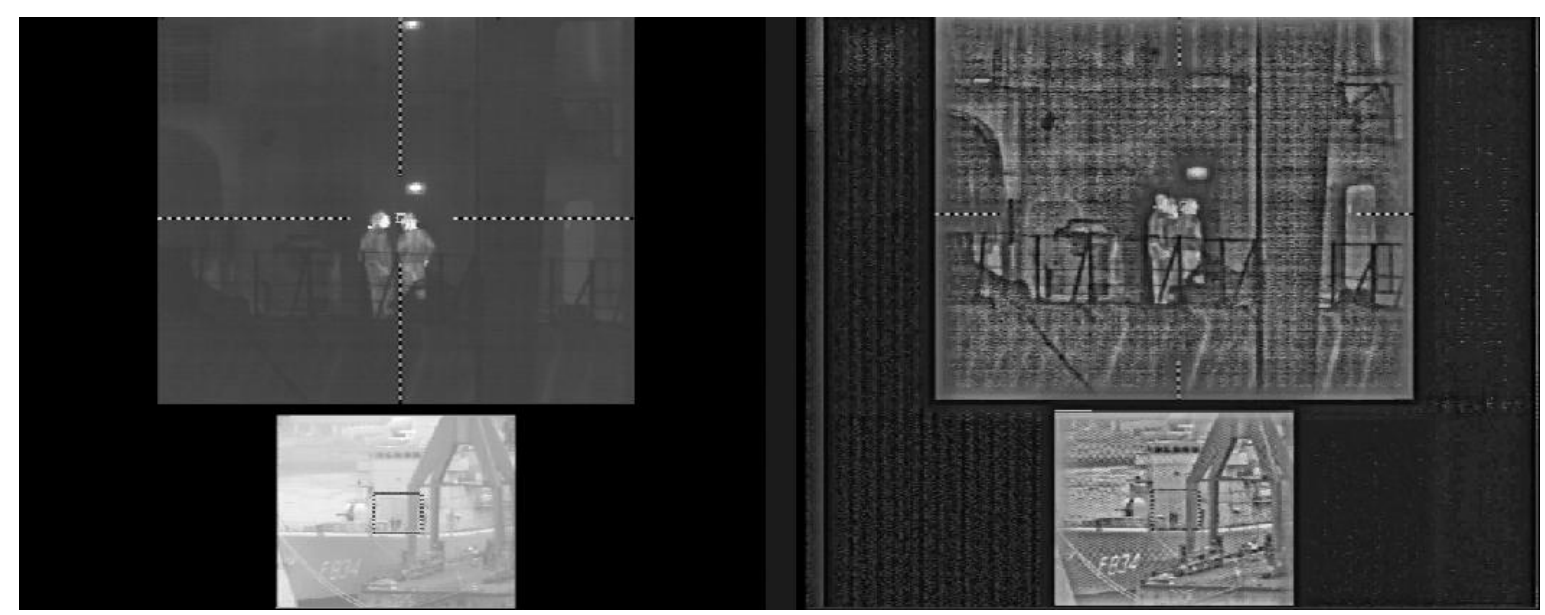

Figure 7: Harbour operations (left original MWIR and (colour) TV, right with enhanced contrast).

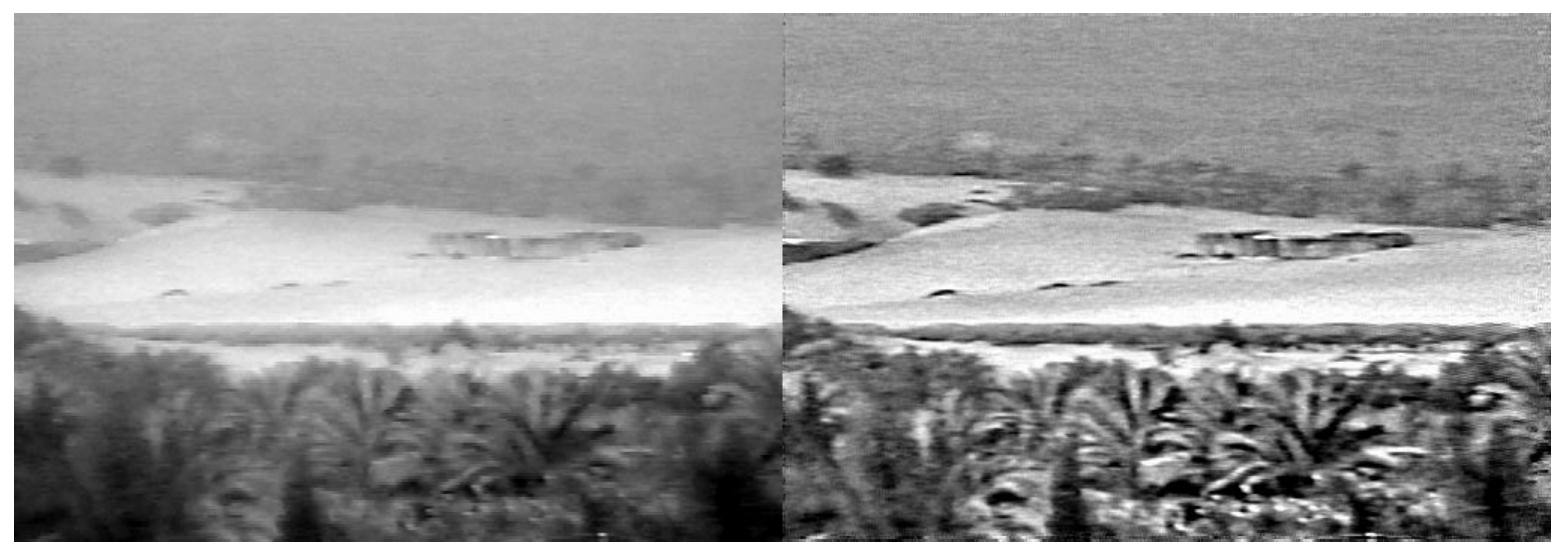

Figure 8: Coastal area during Suez channel passage (left original LLL-TV, right enhanced contrast).
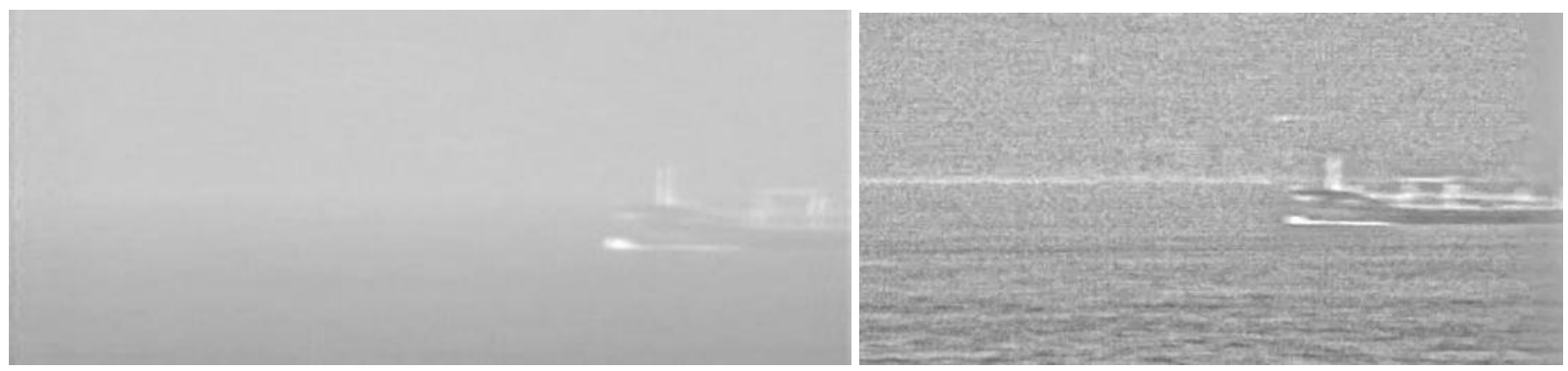

Figure 9: Ship, sea surface and coastal background during passage near Gibraltar (daylight camera left without and right with contrast enhancement).

\subsection{Adaptive sensor management}

The aim of adaptive sensor management is to optimise the process parameters in order to improve the extraction and presentation of target and background data to the end-user, being human operator or weapon system. This can be achieved by interfering with the acquisition, pre-processing, processing and data extraction steps. This, however, is dependent on the tasks of the sensor (or sensor operator), the limitations of sensor and continuous changing condition of target and target background, including active and passive interference. The adaptive sensor management module is well suited for supporting the process, and it therefore plays a central role as Arbiter for all processes in Figure 2. On 
the basis of the outputs of the different algorithm blocks camera settings can be adapted to optimize the processing results.

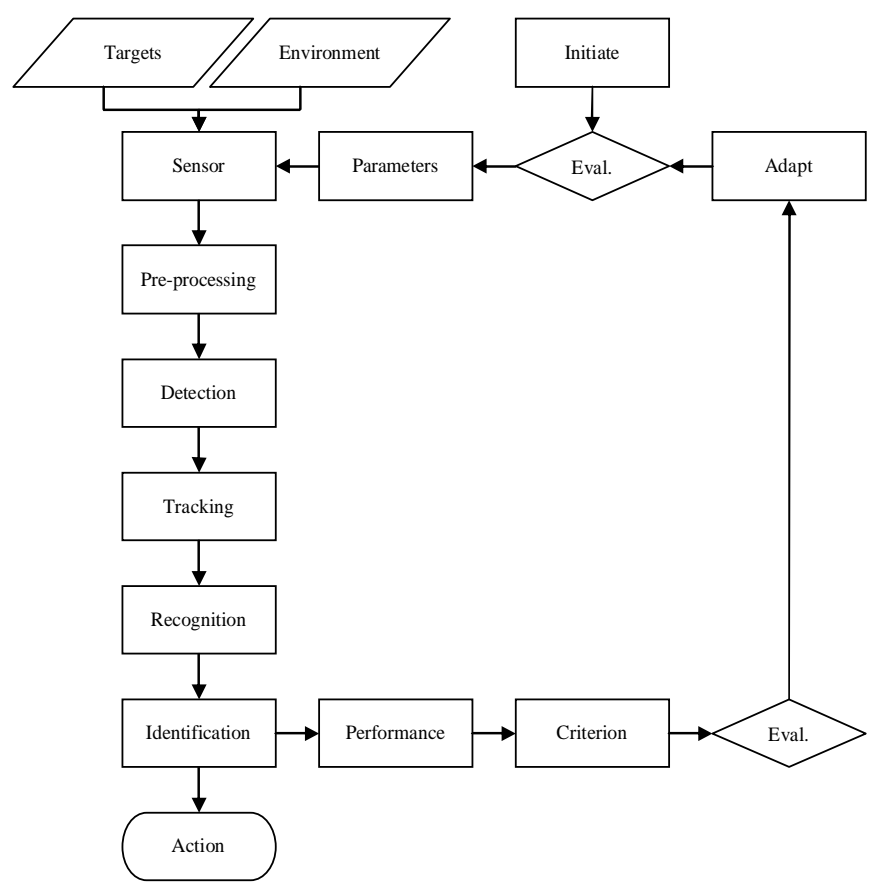

Figure 10: Potential flow diagram of adaptive sensor management of electro-optical sensors (for clarity reasons in this figure shown for identification only; in principle each block from pre-processing to identification should feedback to the sensor in a similar way).

In Figure 10 an example is given of a generic processing scheme with a feedback loop for an autonomous target detection sensor. Data of the target and its surrounding environment are acquired by the sensor, which received its initial parameters via the 'upper' evaluator (Eval.). Next the raw data is converted to the pre-processor to correct data for specific sensor characteristics like bias or interlacing. In the detection process, the signals of potential targets are detected and the position and other features of these potential targets are estimated. The results of the detection process are plots which not only originate from targets in the field-of-view (FOV) of the sensor plots, but also from sensor noise, background clutter and interference (e.g. enemy countermeasures). In the tracking process the target states (position, course and speed) are estimated from the plots provided by the detection process. The tracks are used as input for the recognition process where the target is recognised based on the current estimate and history target features. After recognition identities are assigned to the tracks, e.g. friend, foe, neutral, or unknown. In general each processing block should be provided with the same path of performance evaluation as given in the figure.

Whether all processes are applied to the sensor data depends on the application. In some applications there may be no interest in target recognition or no need to estimate target properties with a tracking process. In some cases the sequence of the processes may be interchanged, e.g. track-before-detect. In this example the identification results are evaluated by means of a performance metric and a criterion. In case the performance of this process does not meet the criterion the initial sensor parameters are changed for the next processing steps. In principle each processing step can have its own self-assessment, validation and parameter estimation techniques. The outputs of these evaluation steps can be fed back into the single sensor processing scheme or converted to another (sensor) processing scheme. Also information to control the self-assessment, validation and parameter estimation techniques can be obtained from another (sensor) processing scheme.

For example an IRST system consisting of two sensors (e.g. SIRIUS ${ }^{8}$ ) operating in respectively 3-5 $\mu \mathrm{m}$ and 8-12 $\mu \mathrm{m}$. Both sensors are used for target detection and would be weighed according to their respective atmospheric transmission. When at a certain moment the atmospheric conditions change, the ratio of the atmospheric transmission of both wavelength bands can change which can give rise to new weighing factors for each sensor. 


\subsection{Evaluation criteria}

Methods such as TOD, MRTD, DMRT and MTDP are laboratory measures that were developed to predict static performance for sensors without sophisticated image enhancement techniques. The present study focuses on their applicability for sensors with static and dynamic image enhancement techniques. Bijl et al. (2006) ${ }^{9}$ have shown that MRTD and DMRT are principally unsuited for dynamic image enhancement techniques, while the MTDP laboratory test yields unexpected pessimistic results for image enhancement. Only the TOD curves are in correspondence with the expected performance improvement.

The final test is a validation against sensor performance for realistic targets. For this purpose, two large sets of static and dynamic imagery were collected and prepared for further processing: one with single hand-held objects and one with two hand-held objects, both at a wide range of distances and at many viewing aspects. In 2007 different types of image processing will be applied to the sets: LACE (Local Adaptive Contrast Enhancement), DSR (Dynamic Super Resolution) and SBNUC (Scene Based Non Uniformity Correction) at different levels. Identification experiments with human observers will be performed on the imagery and the results will be compared with the TOD and MTDP measurements from Bijl et al (2006) ${ }^{9}$. The results will show how well the TOD and MTDP are suited to predict the performance for sensors with image enhancement techniques.

\section{APPLICATIONS}

Our developments have resulted in real-time hardware implementations of algorithms for scene-based non-uniformity correction, stabilisation, contrast enhancement, resolution enhancement and noise reduction. These techniques, that provide an improved image, are the pre-processing stage before the detection and classification process. In the recent past the real-time image enhancement system has been demonstrated on thermal and daylight imagery in operational army environments (compound security) in Afghanistan, airborne UAV (the Sperwer Remotely Piloted Vehicle Image Enhancement) applications, and in maritime environments on-board the Netherlands air-defence and command frigates. The system is improving the situational awareness in these diverse defence operations. In Figure 11 we show the system set-up in Afghanistan where the processing is applied to imagery.

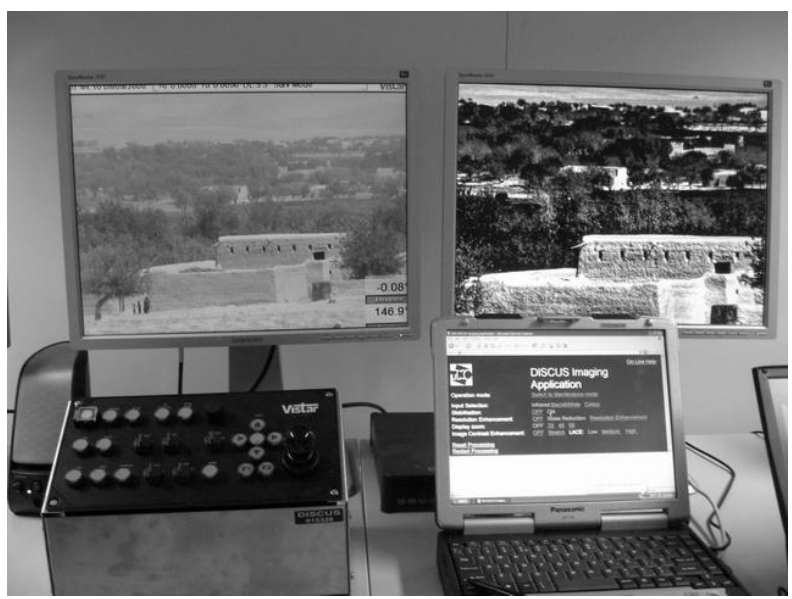

Figure 11: TNO Image Enhancement System operational in Uruzgan, Afghanistan DISCUS (left visible light, right midwave infrared).

The assessment of the performance of these image enhancement techniques in operational scenarios allows us to design new system concepts and develop new system evaluation equipment. These new systems, that make optimum use of the new techniques, can consist of cheaper sensor components with similar performance as more complex sensors without these techniques. Furthermore new developments are based on the feedback from these real-time applications. 


\section{CONCLUSIONS}

We have presented our EO sensor systems programme. This programme is aiming for detection and in particular classification of asymmetric threats in coastal environments. The programme studies system concepts for new types of IRST systems, as well as signal processing to enhance images, to perform detection and classification as well as adaptive sensor management. We have presented some examples of the research. For image enhancement techniques a lot of benefit can be gained in classification over long horizontal paths, when turbulence correction is applied. We have

developed a technique based on our dynamic super-resolution and local contrast enhancement algorithms. The results of these techniques are very promising. These techniques are being exploited to be applied in real-time environments. Examples of these applications are given.

\section{ACKNOWLEDGEMENTS}

The work for this paper was supported by the Netherlands MoD under the programme V602 "Electro-Optical sensor systems". Data recorded during naval operations was distributed to TNO by the Netherlands MoD. The authors acknowledge contributions to the paper by co-workers in the programme V602.

\section{REFERENCES}

1. Schwering, P.B.W., 2006, IRST evaluation methodologies: Maritime infrared background simulator, SPIE Proc. Vol. 6206, 620621, 2006

2. de Vito S., Barani G., Uda G., Mottin E., Clare J.C., Gulley J., Correia B., Davies R., Hickman D., Barron D., Coates P., Benoist P., Salomone F., Tribolet P., Schwering P.B.W., Joppe J.L., RTP 8.2: Intelligent sensorsNew Technology and design concept for Infrared Search and Track Systems, in 'Infrared Information Symposia $2^{\text {nd }}$ NATO-IRIS Joint Symposium', ERIM Vol. 41, No. 4, pp31, London U.K., 25-28 June 1996

3. van den Broek, S.P., Bakker, E.J., de Lange, D.J., Theil, A; Detection and classification of infrared decoys and small targets in a sea background, 2000, SPIE Vol. 4029, 70-80, 2000

4. Defence IQ; Passive Sensing and Targeting 2006; London UK; July 2006

5. Schutte, K., de Lange, D.J.J., van den Broek, S.P., Signal conditioning algorithms for enhanced tactical sensor imagery, SPIE Proc. Vol. 5076, 92-100, 2003.

6. Theil, A., Huizing, A.G., van Heijningen, A.W.P., Single Picture Integration for Territorial Waters Surveillance (SPITS): An initiative to improve situational awareness in littoral waters, MAST (Maritime Systems and Technology) Conference, Nice France, 4-6 September 2006

7. Bosse, E.; Roy, J., The Canada-Netherlands collaboration on multisensor data fusion and other CanadaNATO MSDF activities, Circuits and Systems, 1998. ISCAS apos; 98. Proc. of the 1998 IEEE, May-Jun 1998, Volume 6, Issue 31, 561-564, 1998

8. $\quad$ Knepper, R., Sirius, a long range infrared search and track system, SPIE Proc. Vol. 3061, 578-584, 1997

9. Bijl, P., Schutte, K., Hogervorst, M.A., Applicability of TOD, MRT, DMRT and MTDP for dynamic image enhancement techniques, SPIE Proc. Vol. 6207, 154-165, 2006 